\title{
Alimentation animale et organisation des filières : une comparaison pois protéagineux-lin oléagineux pour comprendre les facteurs freinant ou favorisant les cultures de diversification
}

François Charrier ${ }^{1,2, a}$, Marie-Benoît Magrini' ${ }^{2}$, Aude Charlier ${ }^{3,4}, \mathrm{M}^{\prime}$ hand Fares ${ }^{2}$, Marianne Le Bail ${ }^{3}$, Antoine Messéan ${ }^{4}$ et Jean-Marc Meynard ${ }^{5}$

${ }^{1}$ UR0045 INRA-LRDE (Laboratoire de Recherches sur le Développement de l'Élevage), Quartier Grossetti 20250 Corte

2 UMR1248 INRA-INPT AGIR (Agrosystèmes, agricultures, Gestion des ressources, Innovations \& Ruralités), chemin de Borde-Rouge, BP 52627, 31326 Castanet-Tolosan Cedex, France

3 UMR1048 INRA-AgroParisTech SAD-APT (Sciences pour l'Action et le Développement - Activités, Produits, Territoires), 78850 Thiverval-Grignon, France

4 UAR1240 INRA EcoInnov «Impacts écologiques des innovations en production végétale », 78850 Thiverval-Grignon, France

5 Département INRA SAD (Sciences pour l'Action et le Développement), 78850 Thiverval-Grignon, France

Reçu le 24 avril 2013 - Accepté le 15 mai 2013

\begin{abstract}
Résumé - Le processus d'intensification des élevages, entamé dans les années 1970, s'est accompagné d'un développement croissant du recours aux aliments dits «composés », fabriqués à partir de différentes matières premières pour la plupart d'origine végétale. Les pratiques de la formulation des fabricants d'aliments composés, fondées sur une très forte substituabilité des matières premières, génèrent une concurrence très forte entre ces commodités. Dans un tel contexte de marché, la valorisation de certaines espèces repose sur des mécanismes permettant de contourner ou de s'affranchir de cette concurrence, mais dont l'efficacité reste fortement dépendante de l'organisation des acteurs au sein des filières. À partir de la comparaison des filières pois et lin, nous montrons ainsi qu'au-delà de la reconnaissance de propriétés nutritionnelles spécifiques pouvant valoriser différemment certaines espèces, la coordination des acteurs le long d'une filière est déterminante dans la diffusion des incitations économiques et informationnelles nécessaires à une orientation des agriculteurs en faveur d'espèces de diversification d'intérêt agro-écologique.
\end{abstract}

Mots clés : Nutrition animale / formulation / coordination / marché / pois protéagineux / lin oléagineux

\begin{abstract}
Feed and organization of chains of production: a comparative analysis of protein pea and linseed agricultural chains. The intensification process of livestock farming, connected to a growing utilization of compound foods manufactured from several types of crop raw materials, has started in the 1970's. Formulation, as a central strategy for manufacturers of compound feedingstuffs, is based on the high substitutability between raw materials, which generate a high level of competition between these commodities. In this market conditions, commercializing certain crop productions is founded on mechanisms allowing bypassing this competition, but whose effectiveness is highly dependent on the stakeholders' organization within the food chain. By presenting a comparative analysis of protein pea and linseed agricultural chains, we demonstrate that, beyond valorizing specific nutritional properties of certain species, the coordination of stakeholders throughout the agrofood chain is a key condition to share the informational and economical incentives in order to promote crops of agro-ecological interest.
\end{abstract}

Keywords: Animal nutrition / formulation / coordination / market / protein pea / linseed

\section{Introduction}

Les deux-tiers des surfaces en grandes cultures sont aujourd'hui destinées au marché de l'alimentation animale. Depuis les années 1970, le développement des aliments dits

\footnotetext{
a Correspondance : charrier@corse.inra.fr
}

« composés » dans les schémas d'alimentation des animaux d'élevage a permis le développement de débouchés industriels supplémentaires de nombreuses espèces végétales. Une place croissante a été donnée aux tourteaux issus de l'industrie agro-alimentaire (en particulier le soja) et des agro-carburants (comme le colza par exemple). Ils occupent aujourd'hui environ $30 \%$ des formules d'aliments composés. Tandis que les 
céréales (et leurs coproduits) représentent près de $60 \%$ des taux d'incorporation, une place importante confortée par les normes d'incorporation dont elles bénéficient. En revanche, les graines oléo-protéagineuses occupent une place relativement congrue : moins de $2 \%$ des taux d'incorporation (Fig. A.1, Annexe). Alors que ces espèces peuvent présenter des intérêts nutritionnels, mais également des intérêts agro-écologiques majeurs en termes de diversification des cultures (voir D403 de ce numéro d' $O C L$ ).

La diminution de certaines espèces dans la sole française a été renforcée par un désengagement progressif de l'État dans des mécanismes publics de régulation des marchés, alors que les rapports de compétitivité entres espèces ont considérablement évolué ces dernières décennies. Les rendements et les cours des céréales ont connu une progression importante, renforçant un rapport de marché défavorable aux graines protéagineuses qui les ont rendus moins attractives pour les agriculteurs. Le développement des tourteaux de soja à l'international et d'autres tourteaux d'oléagineux d'origine métropolitaine (colza, tournesol) - dont les profils nutritionnels sont supérieurs aux graines protéagineuses pour le complexe «protéines » des formules - ont renforcé l'attraction des FAC envers ces espèces. En conséquence, en l'absence du maintien des «plans protéines » et de relais par les marchés, la culture des légumineuses à graines telles que le pois, reste insuffisamment attractive pour les agriculteurs. Il en est de même pour la culture du lin oléagineux dont le niveau de rendement au regard de son prix de marché est insuffisamment compétitif au regard des autres espèces privilégiées dans les formulations. Cependant, alors que le pois reste considéré comme une commodité comparée aux autres matières premières, le développement d'un marché de niche en alimentation animale a ouvert de nouvelles opportunités pour le lin depuis le début des années 2000. La création d'une nouvelle filière sur ce marché, fondée sur la valorisation nutritionnelle de la graine de lin pour ses propriétés lipidiques (richesse en oméga 3), a permis de redéployer les surfaces de cette culture.

Nous proposons d'exposer ici comment la structuration d'une nouvelle filière a permis de relancer la culture du lin dans certaines régions, alors qu'à l'inverse les surfaces en pois tendent à poursuivre leur baisse. L'analyse comparative de ces deux filières, pois et lin, vise à montrer qu' au-delà de la reconnaissance de propriétés nutritionnelles spécifiques valorisant différemment ces deux espèces sur ce marché, la coordination des acteurs le long d'une filière reste déterminante dans la diffusion des incitations économiques et informationnelles nécessaires à une orientation des agriculteurs en faveur d'espèces de diversification d'intérêt agro-écologique.

L'article est structuré comme suit. Dans une première partie, nous rappelons le fonctionnement du marché des aliments composés pour la nutrition animale, afin de comprendre les difficultés rencontrées par les graines oléo-protéagineuses françaises pour être valorisées dans ce marché. Dans une seconde partie, nous reviendrons sur les difficultés spécifiques du pois sur ce marché, notamment sur l'absence de coordination de cette filière, puis évoquerons la question de l'avenir de cette filière. Nous terminerons par l'analyse de la filière lin, révélant une capacité de valorisation et de structuration différenciée. En conclusion, nous reviendrons sur les enjeux majeurs qui peuvent être établis à partir de cette comparaison pour favoriser la diversification des cultures.

\section{Le marché des aliments composés : une concurrence difficile pour les graines d'oléo-protéagineux}

L'objectif de la formulation est de mettre à disposition des animaux, de façon quotidienne, l'ensemble des ingrédients satisfaisant leurs besoins. Le principe général de la formulation des aliments composés consiste à calculer, dans une conjoncture donnée de prix, pour chaque type d'aliment, le pourcentage d'incorporation de chaque matière première permettant d'obtenir la composition nutritionnelle souhaitée, à un coût minimum (Gueguen et al., 2008). La formulation fait donc le lien entre la demande et l'offre, en mettant en regard la production agricole avec les besoins nutritionnels des élevages et leurs performances.

\subsection{La formulation : un outil industriel optimisé}

Évoluant au fil des progrès technologiques, des connaissances sur la nutrition animale et sur la qualité des productions végétales, la formulation permet aux fabricants d'aliments d'utiliser une gamme de plus en plus large de matières premières. Pour une recette alimentaire donnée, les formulateurs peuvent combiner une grande diversité de matières premières, au profil nutritionnel complémentaire dans la formule. La formule est raisonnée à partir d'une combinaison nutritionnelle à atteindre (composition en glucides, lipides, phosphore...) et ne dépend pas de la nature des matières premières (tourteau de soja, graines...). C'est le principe de «nutriment anonyme » : la recette s'exprime sous la forme d'une combinaison de nutriments pouvant provenir de différentes matières premières. La qualité de la recette est donc moins dans la nature des ingrédients qui la composent, que dans le niveau des caractéristiques nutritionnelles et alimentaires qu'elle permet d'atteindre. Le formulateur privilégie ainsi une représentation fonctionnelle des matières premières pour ajuster les approvisionnements, en jouant sur la grande diversité des matières premières utilisables et leur haute substituabilité (Lapierre et Pressenda, 2002 ; Lapierre, 2004 ; 2005).

Mais, l'intérêt des matières premières pour les FAC dépend aussi des conditions d'approvisionnement et de leur disponibilité. La pratique de la formulation prend en effet en compte de nombreux paramètres sur les conditions d'approvisionnement (prix de marché, capacité de stockage, coûts de transport, régularité, stabilité de la qualité, fiabilité du fournisseur, etc.). La formulation relève donc d'une véritable logique d'optimisation de l'outil industriel. Les formules sont le résultat d'une interaction étroite entre le formulateur (davantage connecté aux besoins des éleveurs) et l'acheteur de l'usine (davantage connecté au marché et aux conditions d'approvisionnement). Si la notion de «prix d'intérêt» d'une matière première (prix de marché au-dessus duquel une matière première devient moins intéressante qu'une autre par rapport à son apport nutritionnel) est souvent utilisée, il paraît plus juste 


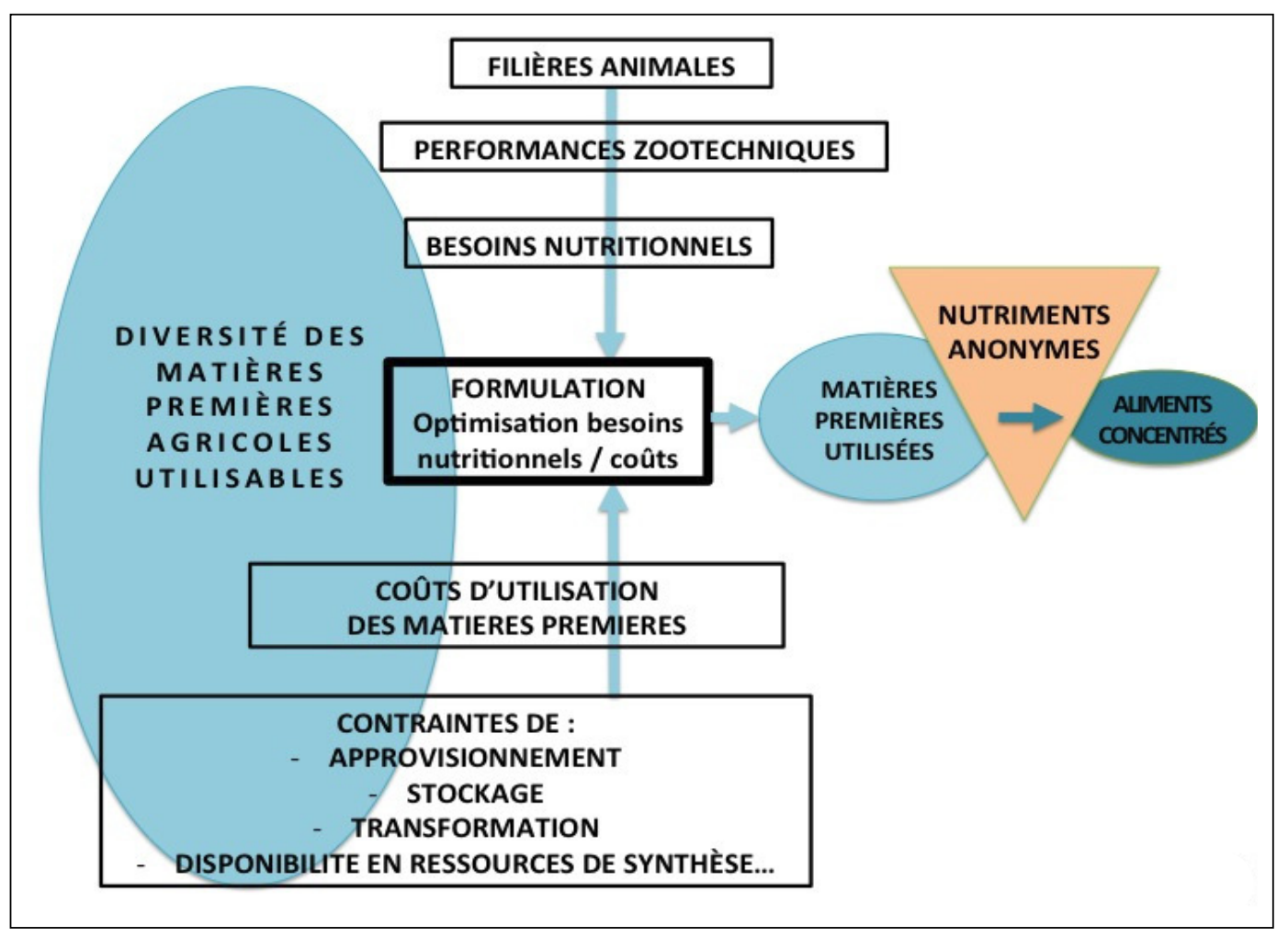

Fig. 1. Schématisation de la pratique de la formulation en alimentation animale (d'après Lapierre et Pressenda, 2002).

de parler de « prix de substitution », notion prenant en compte l'ensemble des conditions économiques liées à l'approvisionnement, au stockage et à la transformation/assemblage, qui orientent les choix de matières premières. Il apparaît alors que, si le prix de marché de la matière première, qui peut contribuer jusqu'à $70 \%$ au coût de l'aliment ${ }^{1}$, est un poste sur lequel les FAC ont peu de marge de manœuvre sur sa fixation, les conditions d'approvisionnement (impactant les coûts de transaction ${ }^{2}$ ), les conditions de stockage (disponibilité des cellules), de transformation technologique, sont autant de leviers sur lesquels les FAC peuvent jouer dans l'optimisation économique de leurs formules d'aliments. En ce sens, la planification des activités de stockage, puis de transformation et de calibrage des outils industriels, orientent les stratégies d'approvisionnement des FAC pour réduire les coûts de fabrication des aliments composés au regard du cours des matières premières ${ }^{3}$. Le marché des matières premières pour l'alimentation des animaux fonctionne donc selon une logique «spot» qui repose sur cette substituabilité des matières. En ce sens, le développement de la formulation a profondément modifié l'organisation du marché de l'approvisionnement des matières premières (Sauvant, 2004).

\footnotetext{
${ }^{1}$ Coop de France NA, 2011 (présentation de V. Bris du 29/09/2011).

2 Par coût de transaction on entend l'ensemble « des coûts de recherche et d'information, des coûts de négociation et de décision, des coûts de surveillance et d'exécution » (Coase, 2005, pages 23-24).

${ }^{3}$ Ceci se traduit par des évolutions d'indices IPAMPA et IPAA différenciées.
}

\subsection{Une compétition défavorable aux graines d'oléo-protéagineux}

L'alimentation des élevages se caractérise de plus en plus par un recours important au marché, tout particulièrement pour les élevages de volailles dont les aliments composés peuvent représenter jusqu'à $80 \%$ de la ration (Lapierre, 2011 ; Dronne, 1995; Sauvant, 2004). Les matières premières utilisées par l'industrie française de la nutrition animale proviennent aux trois-quarts de l'hexagone. Certaines matières premières n'étant pas disponibles en quantité suffisante, les fabricants s'approvisionnent sur les marchés extérieurs. C'est le cas des tourteaux de soja qui proviennent essentiellement d'Amérique du Sud. Le Brésil et l'Argentine représentent respectivement $68 \%$ et $7 \%$ des importations françaises de tourteaux de soja (SNIA, 2012 $2^{4}$; MAAF, 2012 ${ }^{5}$ ).

Malgré la large gamme de matières premières utilisables et les différents leviers de l'outil industriel sur lesquels les FAC peuvent jouer, le schéma d'approvisionnement des FAC est relativement standardisé autour du couple tourteaux-céréales, et plus précisément du couple soja-blé au regard du complexe protéines-énergie (Fig. 2). Cette standardisation s'explique d'abord par une tendance à la simplification des formules dans une logique d'économie d'échelle et de massification des approvisionnements. Ainsi, la plupart des usines étant

\footnotetext{
${ }^{4}$ ISGA - Ambassade du Brésil 28 juin 2012. Présentation du SNIA.

5 MAAF, 2012. Panorama de IAA 2012-Fiche Sectorielle. Sous Classe $10.91 \mathrm{Z}$ : fabrication d'aliments pour animaux.
} 


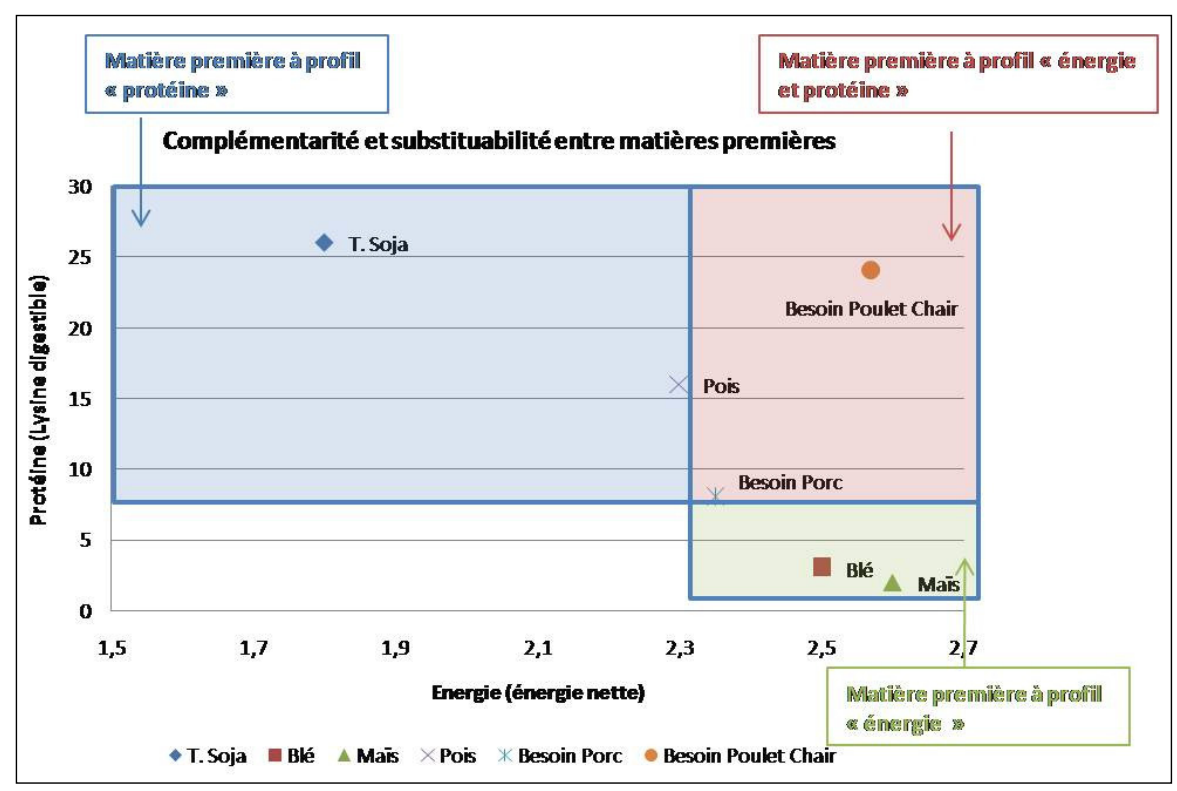

Fig. 2. Complémentarité et substituabilité de matières premières selon leur profil nutritionnel et les besoins d'élevages en porcs et poulets de chair (d'après Lapierre, 2011).

polyvalentes $(80 \%$ des usines fabriquent des aliments pour différents types d'élevages $\left.{ }^{6}\right)$, les FAC tendent à privilégier des matières premières également polyvalentes (i.e. pouvant être utilisée dans différentes gammes d'aliments). Les matières premières entrant dans la filière la plus importante pour le chiffre d'affaires d'une usine tendent donc à être utilisées plus fortement dans les autres filières d'élevage qu'elle fournit, comparativement à d'autres usines qui se seraient plus spécialisées sur d'autres filières. Malgré la segmentation du marché en aval (i.e. la diversification de la gamme d'aliments), la recherche d'économie d'échelle dans le coût de fabrication des aliments favorise finalement une simplification des formules, et donc des schémas d'approvisionnement.

Pour autant, si les productions animales «standards» permettent aux formulateurs de jouer davantage sur la substituabilité des matières premières (cahiers des charges moins exigeants sur la composition des aliments) et de réaliser ces économies d'échelles, certaines filières visent des critères de production (en quantité ou en qualité) qui contraignent les formulateurs à privilégier une matière première plutôt qu'une autre. L'analyse de l'organisation des filières du pois protéagineux et du lin oléagineux peut alors permettre de comprendre comment des espèces peuvent être différemment valorisées sur ce marché de la nutrition, offrant ainsi des débouchés différents pour soutenir leur mise en culture dans le cadre d'une diversification des assolements.

\section{Le pois dans une filière faiblement coordonnée : quel avenir?}

Traditionnellement destiné au marché de l'alimentation animale, le pois protéagineux a bénéficié d'importants soutiens

\footnotetext{
${ }^{6}$ D'après le Céréopa (Centre d'étude et de recherche sur l'économie et l'organisation des productions animales).
}

des politiques agricoles pour renforcer sa compétitivité, tout particulièrement après l'embargo sur le soja des années 1970. Pour autant ces différents plans de soutien n'ont pas permis de relancer durablement la production de pois, dont les surfaces sont en chute depuis le début des années 1990. Son insuffisante compétitivité sur ce marché et l'absence de coordination de cette filière interroge sur l'avenir de cette espèce pour ce débouché.

\subsection{Le pois : une commodité parmi d'autres...}

Le pois protéagineux a un profil nutritionnel qualifié « d'intermédiaire », au regard de sa composition énergétique et protéique. Principalement concurrencé dans les formules par le couple tourteau de soja-blé, matières premières respectivement très concentrées en protéines et en amidon, le pois est hautement substituable. D'autant plus que ces deux autres commodités sont très présentes sur le marché, comme nous le rappelions ci-avant. Le développement récent d'autres coproduits de l'industrie agro-alimentaire, mais également de l'industrie de l'éthanol (DDGS ${ }^{7}$ ) et des agrocarburants a élargi la gamme des matières premières (tourteau de colza, tournesol...) substituables et renforcé le défaut d'attractivité du pois.

De plus, pour ne pas alourdir les coûts logistiques, les nutriments de synthèse occupent une place croissante pour remplacer certaines matières premières, dont les conditions d'approvisionnement ne répondent plus aux volumes seuils demandés par les FAC. Ainsi, si un intérêt majeur du pois réside dans sa teneur en lysine, le développement de la fabrication de lysine de synthèse (et plus généralement des acides aminés de synthèse) affecte son attractivité auprès des FAC.

${ }^{7}$ Dried Distillers Grains with Solubles : coproduits des végétaux utilisés pour la production d'éthanol par l'industrie des agrocarburants. 


\section{2 ... perçue comme insuffisamment compétitive}

Le pois souffre d'un rapport de compétitivité défavorable par rapport aux autres matières premières. Si les problèmes agronomiques affectant le rendement du pois (notamment le pathogène Aphanomyces euteiches) sont fréquemment mis en avant par les acteurs, le problème de fond semble davantage concerner la substituabilité du pois par rapport aux autres matières premières utilisées dans les formules. La compétitivité du pois est en effet fortement dépendante du mode d'organisation de ce marché, dont nous avons esquissé les traits principaux dans la Section 1. Or, de nombreux facteurs liés à l'organisation du marché de l'alimentation animale lui sont défavorables.

D'abord, la concentration géographique des FAC dans les zones d'élevage pénalise le recours à des productions végétales dispersées sur le territoire. En effet, les usines se situant au plus près de leurs clients (les élevages), la demande est généralement concentrée dans certaines régions, comme en Bretagne fortement spécialisée en élevage porcin. La logistique étant un paramètre important dans la démarche d'optimisation de la formulation, la localisation de l'offre peut donc poser des problèmes d'accessibilité des usines aux matières premières, tout particulièrement lorsque leur culture est dispersée et en faible quantité sur le territoire national. Ce problème d'accessibilité se pose particulièrement pour le pois, et plus généralement pour les légumineuses à graines qui représentent moins de $2 \%$ de la SAU. Alors que la proximité des ports maritimes en Bretagne facilite des approvisionnements massifs en tourteau de soja, au détriment d'autres matières premières qui lui sont substituables, mais qui sont plus dispersées sur le territoire entre des silos de plusieurs fournisseurs. L'approvisionnement pour des matières dispersées géographiquement augmente donc les coûts de transaction. Quant au blé, autre concurrent du pois, il est disponible et accessible en grande quantité dans les silos des coopératives bretonnes. Ensuite, la démultiplication des cahiers des charges (plus de 600 cahiers selon le Céréopa) contraint à choisir des formules globales facilement adaptables et où le pois trouve plus difficilement sa place, surtout dans les cahiers des charges des volailles sous label qui imposent $65 \%$ à $85 \%$ de céréales.

Par ailleurs, à défaut de contrats, l'impossibilité de s'approvisionner à termes, c'est-à-dire au-delà de la fin de l'année, où les volumes sont très faibles, renforce le manque de visibilité sur l'offre du pois pour les FAC. Cependant, ce relatif manque de visibilité pourrait être pallié par une politique commerciale offensive des organismes stockeurs (OS) sur l'alimentation animale. Or, d'une part, ce secteur est considéré comme une variable d'ajustement au vu d'une démultiplication des coproduits qui ne favorise pas la mise en avant du pois comme une matière première de qualité. D'autre part, l'émergence récente de débouchés en alimentation humaine, mieux valorisés (par exemple, les pois jaunes à l'export), réoriente la politique commerciale des OS en faveur de ce marché. Le prix du pois devient alors intéressant pour les FAC dans le cas où l'OS, en fin de campagne, doit libérer une cellule de stockage et décide de «se débarrasser» du pois invendu. Le FAC n'a donc pas intérêt in fine à s'engager sur un prix ferme en début de campagne, n'incitant donc pas les OS à mettre en avant le pois comme une espèce d'intérêt pour ce marché. Il n'y a donc pas de coordination forte entre l'amont et l'aval pour cette espèce.

Cette faiblesse des liens de coordination entre l'amont (production végétale) et l'aval (FAC et élevages, mais également autres débouchés), que ce soit en termes de modes de transaction (contrats) ou d'échanges d'information technicoéconomique sur le pois, renforce le manque de compétitivité du pois. En effet, la dynamique de l'amont de la filière en termes d'amélioration variétale, d'essais agronomiques et de communication active sur cette culture auprès des agriculteurs ${ }^{8}$ ou des utilisateurs est très dépendante de celle de l'aval (Meynard et al., 2013). Ce manque de coordination semble ainsi contribuer au désintérêt progressif pour le pois.

\subsection{Relancer la compétitivité du pois par une meilleure coordination de la filière?}

Derrière les défauts de compétitivité du pois dans les formules pour l'alimentation animale, apparaissent donc des problèmes de coordination majeurs au sein de la filière. Les incitations économiques et informationnelles ne se diffusent pas auprès de 1 'ensemble des acteurs, et notamment des agriculteurs. Aussi, une meilleure coordination horizontale (amontaval), mais aussi verticale, serait une condition essentielle à la production et une utilisation de pois protéagineux à une plus large échelle. En effet, les freins à la production, et surtout à la valorisation de cette culture, peuvent être adressés de manière partagée entre les acteurs, notamment en ce qui concerne les questions d'ordre logistique comme la capacité de stockage (adaptée aux volumes de pois) et la massification des approvisionnements (« re-concentrer » l'offre géographiquement dispersée), ainsi que les questions de mise en marché comme la capacité d'anticipation des volumes par les FAC. La contractualisation des surfaces serait un mécanisme potentiellement efficace pour adresser ces questions, et permettre au pois de se démarquer de la concurrence du marché spot. Mais pour cela, il conviendrait de mettre en avant une qualité spécifique du pois «légitimant» son besoin de production. À défaut d'une reconnaissance nutritionnelle spécifique dans son usage pour l'alimentation animale, la mise en avant de qualités environnementales pourrait renforcer sa production. D'abord, la mise en avant de services écosystémiques spécifiques, notamment au regard des enjeux de fertilisation et de réduction des pollutions par les nitrates, favoriserait une meilleure diffusion des informations sur la culture de pois protéagineux (références technico-économiques) et servirait la durabilité d'autres productions végétales. Ensuite, les filières d'élevage peuvent être amenées à jouer un rôle dans leur contribution à la réduction des GES par leur choix d'alimentation et d'approvisionnement. Un repositionnement du pois dans ces choix de production permettrait ainsi d'établir une contribution non négligeable de ces acteurs à ces différents enjeux environnementaux (fertilisation, changement climatique...), nécessitant une meilleure coordination entre filières, puisque le partage d'enjeux incite à la coordination des actions.

\footnotetext{
8 Comme l'étude Meynard et al., 2013 le met en avant, une meilleure communication sur des valeurs de références relatives aux bénéfices pluri-annuels de la diversification des grandes cultures, valoriserait mieux la culture du pois dans les rotations.
} 
Par ailleurs, sur le plan de la nutrition, l'émergence d'un nouveau débouché à plus haute valeur ajoutée en alimentation humaine ouvre des opportunités intéressantes pour cette culture (Géhin et al., 2010). Si ce nouveau marché « détourne» les volumes de pois de l'alimentation animale, il est susceptible de générer un intérêt croissant de certains opérateurs pour cette culture, voulant investir dans la filière pour lever différents verrous. Ce positionnement des acteurs rendrait le pois protéagineux «moins substituables », car recherché spécifiquement par des industriels ciblant des marchés spécifiques. Faisant l'hypothèse que le développement du pois pour l'industrie agro-alimentaire est susceptible de générer des coproduits du pois pour l'alimentation animale, le développement de nouveaux débouchés est susceptible de contribuer in fine à une valorisation globale de l'espèce plus intéressante que sa seule valorisation sur le marché des FAC. Afin de répondre à cette demande, ceux-ci chercheraient à sécuriser des approvisionnements réguliers en pois, et donc à mettre en place des mécanismes incitatifs auprès des agriculteurs, tels des contrats à prix garanti et indexés. Par conséquent, la coordination des acteurs s'en trouverait renforcée, comme dans le cas du lin oléagineux dans la filière Bleu-Blanc-Cœur.

\section{Lin oléagineux : une filière coordonnée pour un marché de niche}

Par le passé, le lin oléagineux a été essentiellement considéré comme une culture d'opportunité, dont l'implantation des surfaces a suivi les évolutions de la PAC dans les années 1990 et les progrès en sélection variétale. Plus récemment dans les années 2000, le développement d'un marché de produits animaux sous label, valorisant les propriétés lipidiques de la graine de lin, a abouti à la construction d'une filière fortement coordonnée qui a relancé cette culture.

\subsection{L'émergence d'une propriété nutritionnelle spécifique : le rôle des oméga 3}

À partir des années 1990, est mis en évidence l'effet d'une alimentation animale riche en oméga 3 sur la qualité des produits laitiers (Brunschwig et al., 2010) et carnés (Hurtaud et al., 2010; Razminowicz et al., 2008; Noblet et al., 2008; Musela et al. 2009; Mourot, 2009). Puis un lien est établi entre cette alimentation des animaux et la nutrition humaine, à la fin des années 1990 et au début des années 2000, par des études cliniques du CERN et de l'INRA (Weil et al., 2001; Weil et al., 2002). L'amélioration du profil lipidique des animaux contribue à celle de l'homme, notamment dans la prévention des maladies cardiovasculaires. Ce nouveau savoir nutritionnel offre des opportunités pour le développement de schémas alimentaires composés de plantes à haute teneur en oméga 3.

Le marché des produits animaux riches en oméga 3 se développe donc à partir des années 2000, sous le label privé BleuBlanc-Cœur (BBC) fédérant les élevages respectant une alimentation animale riche en oméga 3. La stratégie de la filière repose alors sur la différenciation des produits sur le marché de l'alimentation humaine, par la mise en avant des propriétés nutritionnelles de ces produits, reconnues par les autorités publiques. En effet, le label BBC bénéfice du label public « filière nutrition » défini dans le cadre du Programme National Nutrition Santé (PNNS). Par ce programme, l'État s'engage à soutenir les filières qui s'engagent à améliorer le profil nutritionnel des produits alimentaires. De ce fait, à la différence des cahiers de charges des autres labels officiels (tels que AOC, Label Rouge... ) qui ne définissent qu'une obligation de moyens, les cahiers des charges de la filière BBC imposent également une obligation de résultats (teneur en oméga 3 des produits finis). Pour répondre à cette «contrainte», les éleveurs cherchent donc à s'approvisionner en aliments riches en oméga 3.

La graine de lin, source végétale la plus importante en acides gras polyinsaturés en oméga 3 (acide alpha-linoléique), devient alors une matière première d'intérêt pour les filières développant de tels produits. Les problèmes fonctionnels pour l'utilisation de la graine de lin dans les rations, sont dépassés par le brevetage d'une technologie de thermo-extrusion ${ }^{9}$ par un FAC, Valorex. Cette technologie permet d'éliminer les facteurs antinutritionnels (par cuisson) et d'améliorer l'accessibilité à l'huile (riche en oméga 3) et à l'amidon des graines, tout en préservant les protéines (Poncet et al., 2003). Ce procédé de transformation permet notamment d'améliorer la digestibilité de la graine. Alliant les effets bénéfiques de la thermoextrusion et de la teneur en ALA (acide alpha-linoléique) du lin, les aliments proposés par Valorex se différencient sur la base d'une amélioration de certaines performances zootechniques ${ }^{10}$ et d'une qualité nutritionnelle améliorée des produits animaux destinés à l'alimentation humaine. L'approvisionnement de cet industriel en graines de lin devenant alors un enjeu stratégique pour son activité et le fonctionnement de l'ensemble de la filière, une forte coordination des acteurs est mise en œuvre.

\subsection{Coordination amont-aval pour garantir un approvisionnement de la filière}

La différenciation par la qualité sur le marché des produits animaux de la filière $\mathrm{BBC}^{11}$ créée par Valorex repose aussi sur la traçabilité des produits. Pour faire face à ses besoins, Valorex développe une politique de contractualisation pour sécuriser et garantir la qualité de ses approvisionnements en lin. Les contrats de production entre l'industriel et les agriculteurs (par l'intermédiaires des coopératives) intègrent une clause de respect d'un cahier des charges précis (variétés autorisées, recommandations sur les itinéraires techniques, interdiction de

\footnotetext{
${ }^{9}$ Brevet TradiLin 2006.

${ }^{10}$ Plusieurs effets reconnus ont fait l'objet de différentes publications scientifiques, les plus avancés sont rappelés par Thévenin (2006) : augmentation de la production de lait de 4 à $6 \%$; baisse du risque d'acidose ; augmentation des acides gras polyinsaturés dans le lait; meilleure fécondation de $10 \%$ en première insémination. .

11 BBC (Bleu-Blanc-Cœur) est une association loi 1901, créée à l'initiative de Valorex, dont les adhérents s'inscrivent dans la démarche de valorisation de produits enrichis en oméga 3, selon la charte de l'association, et les cahiers des charges établis pour chaque filière.
} 
certains produits phytosanitaires, suivi et documentation des opérations à la parcelle, étiquetage des lots...).

Afin d'inciter les agriculteurs à insérer le lin dans leur système de culture, le contrat, sur une période de un à trois ans, repose sur une indexation du prix du lin sur le cours des cultures dominantes dans la région (colza, blé). Si les types de contrats sont variés d'un bassin de production à un autre, le prix de vente est couramment fixé selon un «tunnel de prix », lié au cours des grandes cultures présentes dans l'assolement de l'exploitation, l'objectif étant de garantir une marge à l'hectare équivalente à la marge à l'hectare du colza ou du blé. La fixation de ce tunnel (prix minimum et prix maximum de vente) est le fruit d'une négociation annuelle entre l'industriel et les producteurs. Ainsi, $80 \%$ des surfaces de lin oléagineux destinées à ce FAC sont contractualisées et se situent majoritairement dans le bassin de production de l'industriel (grand ouest). Le contrat comprend une rémunération supplémentaire selon la qualité des graines relative à la teneur en oméga 3. Cette grille de rémunération est renégociée régulièrement entre les acteurs de la filière, en fonction des évolutions constatées dans la production et de la progression des connaissances sur l'impact des interactions environnement/plante sur la richesse en oméga 3.

Par ce mécanisme de coordination, la filière se démarque du marché «spot» de l'alimentation animale où la graine de lin oléagineux devient une matière première spécifiquement recherchée par la filière. En outre, l'organisation de cette contractualisation facilite la diffusion de connaissances et des incitations économiques pour favoriser la culture du lin auprès des agriculteurs. Si l'organisation de cette coordination a un coût, la vente des produits sous le label BBC génère une valeur ajoutée supplémentaire qui permet de financer cette coordination aval-amont. En effet, les produits vendus sous ce label sont plus chers de 5 à $10 \%$ par rapport aux produits similaires «standards», ce qui permet à l'industriel de vendre ses aliments à un prix plus élevé, et donc de financer son fonctionnement. Cette forte coordination donne également plus de lisibilité aux acteurs de la filière pour investir dans de nouvelles infrastructures et outils de production.

Pour autant, la conjoncture sur les marchés agricoles apporte une limite importante au modèle : la concurrence au sein des assolements, dans un contexte de prix élevés des cultures dominantes à plus haut rendement (blé, colza, maïs...) peut limiter la négociation sur le plafond du tunnel. Aussi malgré ce dispositif incitatif, l'industriel, dont les besoins sont estimés à $50000 \mathrm{t}$ de graines par an, importe encore aujourd'hui la moitié de son approvisionnement, principalement depuis le Royaume-Uni, où 6500 ha sont sous contrat. Valorex projette aussi de développer la culture du lin dans d'autres bassins de production français que le bassin grand ouest.

\subsection{La coordination par un réseau d'acteur et une association « tête »}

Afin d'assurer une coordination efficace des différents maillons de la filière produisant des produits animaux issus d'élevage ayant reçu une alimentation enrichie en lin, l'association «Bleu-Blanc-Cœur» (BBC) est créée en 2000. C'est une association qui fédère l'ensemble des maillons de la filière, et dont l'organisation suit une vision systémique des filières
Tableau 1. Organisation de l'association BBC en collèges.

\begin{tabular}{c|c}
\hline $\begin{array}{c}\text { Nom du } \\
\text { collège }\end{array}$ & $\begin{array}{c}\text { Maillons de } \\
\text { la filière représentés }\end{array}$ \\
\hline Production végétale & $\begin{array}{c}\text { Semenciers, } \\
\text { coopératives agricoles, } \\
\text { autres organismes collecteurs }\end{array}$ \\
\hline Nutrition animale & $\begin{array}{c}\text { FAC, firmes-services, } \\
\text { ateliers de transformation }\end{array}$ \\
\hline Production animale & $\begin{array}{c}\text { Groupements d'éleveurs, } \\
\text { tous types élevage }\end{array}$ \\
\hline Producteurs fermiers & $\begin{array}{c}\text { Agriculteurs transformant } \\
\text { à la ferme }\end{array}$ \\
\hline Transformateurs & $\begin{array}{c}\text { Laiteries, entreprises de conditionnement } \\
\text { des œufs, de transformation viande, } \\
\text { charcuterie, meuneries, etc. }\end{array}$ \\
\hline Distributeurs & $\begin{array}{c}\text { Chaînes distributeurs, } \\
\text { magasins indépendants, }\end{array}$ \\
\hline Consommateurs & Associations \\
\hline
\end{tabular}

agricoles et des organismes d'appui et d'information. Elle est construite sur la volonté de rassembler tous les maillons de la filière et de renforcer le lien entre les filières végétales et animales. Elle est composée de sept collèges représentant chaque maillon de la filière (Tab. 1) et un collège international, au travers desquels circulent les informations (sur les évolutions variétales, les procédés technologiques, les considérations logistiques, l'analyse des marchés, attentes du consommateur, etc.).

Cette association fait aussi le lien entre les acteurs et les organismes tiers de contrôle de la filière, chargés de certifier les produits et les procédés de production selon les cahiers des charges BBC. Ces audits sont également l'occasion d'échanges entre les acteurs, de conseils, sur les variétés de lin utilisées, les itinéraires techniques, les formules alimentaires conseillées, etc., afin que chaque acteur de la filière puisse disposer d'informations utiles à son activité et que celleci réponde bien aux besoins des autres maillons de la filière.

Soulignons aussi que l'organisation de la filière est caractérisée par des liens forts avec les organismes publics, parapublics et privés du sous-système d'information (associations, bureaux d'études, instituts techniques, instituts de recherche, etc.). Dans les négociations sur les contrats par exemple, il est fréquent que certaines organisations tierces soient associées, comme les chambres d'agriculture. Aussi, certains organismes jouent un rôle de relais entre les agriculteurs des bassins de production et l'industriel. Ces organismes, comme Oléo-Lin, permettent notamment de « re-concentrer» géographiquement une collecte qui tend à se disperser sur le territoire avec l'augmentation des volumes. Alors que cette dispersion géographique apparaît comme un frein important pour l'approvisionnement des FAC bretons en pois protéagineux, ces relais permettent de «contourner» le problème pour l'approvisionnement de l'usine de Valorex. 
Enfin, le déploiement de services conseils est fortement présent sur l'ensemble de la filière. Il fait partie de la stratégie d'incitation à cultiver et à utiliser du lin dans les formules alimentaires. Une filière-Conseil a notamment été créée pour apporter un appui aux acteurs souhaitant s'intégrer dans cette démarche et vendre des produits estampillés BBC. Plus globalement à l'échelle de la filière, un grand nombre d'acteurs ont mis en place un «volet service» (Valorex, les coopératives, les semenciers, les associations de producteurs) : chaque acteur reçoit des formations de la part $d$ 'autres sur les thèmes qui le concernent (itinéraires de culture, stockage, fabrication d'aliments, formules incorporant du lin, organisation de filière...). Ces formations participent de la construction d'un réseau d'acteurs relativement bien coordonnés sur ce marché où la concurrence est grande.

\section{Conclusion : quels défis pour ces deux espèces-filières?}

L'analyse comparative de l'organisation de ces deux filières révèle des dynamiques d'acteurs différentes. Elle met en lumière plusieurs enjeux majeurs pour le développement des surfaces de cultures de diversification, sur lesquels nous proposons de revenir : la coordination des acteurs le long des filières ; les stratégies de différenciation pour contourner le marché spot; la combinaison des débouchés par la création de synergies; l'organisation logistique.

D'abord, la coordination des différents maillons d'une filière, ainsi que les liens de coordination avec les organisations du sous-système d'information, ressort comme essentielle pour diffuser les incitations économiques et informationnelles nécessaires à chaque maillon, dont celui des agriculteurs pour diversifier leur assolement (Fares et al., 2012). Dans cette coordination, la mise en place de contrats à prix garantis contribue à améliorer la visibilité sur les marchés pour les agriculteurs et les FAC. C'est notamment un enjeu majeur pour le pois protéagineux, dont le manque de structuration du marché, caractérisé par une faible information accessible aux agents, pénalise son attractivité. Au contraire, sur le marché du lin oléagineux, les acteurs sont coordonnés par des contrats et des cahiers des charges, favorisant les échanges formels ou informels des informations-clés. Pour autant, c'est un modèle qui trouve ses limites, dans un contexte de prix élevés de cultures concurrentes à plus haut rendement, dans les choix d'assolement des agriculteurs.

Cette coordination est d'autant plus cruciale, que la culture de diversification peut faire l'objet d'une différenciation sur le marché, permettant de contourner la concurrence des autres matières premières sur le marché spot de la plupart des commodités agricoles. Cette différenciation peut permettre de réduire leur rapport de substituabilité aux autres matières premières. La différenciation des produits en aval sur des qualités nutritionnelles et/ou environnementales est un moyen de soutenir ces espèces dans la sole française. Cette différenciation doit aussi reposer sur une signalisation auprès des consommateurs (labels) et une communication importante, comme dans le cas de la filière $\mathrm{BBC}$, pour assurer les débouchés à ces espèces de diversification. L'innovation technologique valorisant ces matières premières est alors un atout supplémentaire, les brevets permettant de protéger ces filières de niches en développement. Si le lin bénéficie d'un affichage nutritionnel, le pois pourrait bénéficier d'un affichage environnemental qui le valoriserait mieux. Dans les deux cas, la signalisation de la qualité et la communication jouent un rôle crucial.

Cette analyse montre aussi, qu'à défaut de la reconnaissance de propriété nutritionnelle spécifique qui rende une espèce végétale moins substituable, les perspectives de valorisation de l'espèce sur le marché de la nutrition animale sont renforcées si elle se présente au statut de coproduit. La notion de coproduit est entendue ici au un sens large comme déchet issu de la transformation, ou lot déclassé ou invendu de marchés agro-industriels. Ainsi, la question de l'émergence de nouveaux débouchés en alimentation humaine pour le pois peut amener à un repositionnement favorable de cette espèce sur le marché de la nutrition animale. Le lin oléagineux peut faire aussi l'objet de différents usages dans l'industrie agro-alimentaire. Organisés sur un schéma industriel pour l'alimentation animale, les acteurs de ces filières sont amenés prendre en compte ces nouveaux enjeux nutritionnels et donc à repositionner leurs stratégies (commerciales, approvisionnement, technologiques...), voire leur cœur de métier. Ces enjeux interrogent d'une part la capacité de ces filières à répondre aux évolutions des attentes des consommateurs, et d'autre part la capacité des acteurs à saisir les synergies potentielles entre chaines de valorisation (mutualisation de technologies et de connaissances sur la matière première). Ces capacités sont fortement dépendantes du degré de coordination des filières pour le partage d'enjeux et de stratégies communes.

Enfin, dans cette organisation de la production, quel que soit le débouché visé, la collecte et la mise à disposition de cultures de diversification pour la transformation, apparaissent particulièrement stratégiques. La logistique reste un poste de coût important. Une meilleure lisibilité des volumes de production, notamment par l'émergence de nouveaux débouchés, peut inciter à investir dans de nouveaux dispositifs, afin d'adapter ces infrastructures à une plus grande diversification des matières végétales. Le marché de l'alimentation animale, confronté à une logique d'économie d'échelle, peut aussi nécessiter une plus grande massification de l'offre via l'organisation de « relais » logistiques sur le territoire.

Pour conclure, cette analyse comparative met bien en avant l'importance de considérer l'ensemble du système agroindustriel pour analyser les freins et leviers à la diversification des cultures. Elle montre aussi que la coordination des filières apparaît comme la clef de voûte des leviers majeurs que nous venons de mettre en évidence pour renforcer des liens entre filières animales et végétales, afin de contribuer à une plus grande diversification d'intérêt agro-écologique de la sole française.

Remerciements. Les auteurs tiennent à remercier l'ensemble des professionnels interrogés lors de cette analyse, et particulièrement Patricia Lecadre et Frédéric Pressenda du Céréopa, pour leurs éclairages sur le fonctionnement du marché de l'alimentation animale. 


\section{Références}

Agreste. 2011. Les matières premières dans les aliments composés pour animaux de ferme en 2009, Agreste Primeur ${ }^{\circ} 258$, mars 2011, $4 \mathrm{p}$.

Agreste. 2012. Surfaces, productions, rendements des céréales, oléagineux, protéagineux, résultats 2011 définitifs, 2012 provisoires. http://www.agreste.agriculture.gouv.fr/thematiques-872/ productions-vegetales-874/grandes-cultures-fourrages-875/ article/surfaces-production-rendements.

Bris V. 2011, Relation animal/végétal : atténuer les impacts de la volatilité pour les productions animales, les propositionsen amont. Sommet de l'élevage, Table ronde URFACAL-Coop de France NA-SNIA; 6 novembre 2011. Clermont-Ferrand, 13 p.

Coase R. 2005. L'entreprise, le marché et le droit, éd. Organisations.

Fares M, Magrini M-B, Triboulet P. 2012, «Transition agroécologique, innovation et effets de verrouillage : le rôle de la structure organisationnelle des filières. ». Cahier d'Agricultures $21: 34-45$

Géhin B, Gueguen J, Bassot P, Seger A. 2010. Répondre aux besoins spécifiques de qualité pour augmenter l'utilisation des légumineuses en transformation industrielle. Innov. Agron. 20 : $115-127$.

Gueguen J, Duc G, Boutin JP, Dronne Y, Munier-Jolain N, Sève B, Tivoli B. 2008. La filière protéagineuse, quels défis? Editions Quae, Paris, p. 160.

Hurtaud C, Agabriel C, Dutreuil M, Rouille B. 2010. Caractérisation de la composition des laits selon les pratiquesd'alimentation dans les principales régions laitières françaises. Rencontres Recherche Ruminants 17 : 381-384.

Lapierre O, Pressenda F. 2002. Adaptation of high protein raw material supply strategies for European livestock feeding. OCL 9 : 86-91.

Lapierre O. 2004. Culture et élevage : quelles relations, quelles synergies ? OCL $11: 261-267$.

Lapierre O. 2005. Système des acteurs et stratégie de formulation. OCL 12 : 217-223.

Lapierre O. 2011. Présentation à la réunion du CNA. Agroparistech, Paris, le 10 février 2011.

Meynard JM, Messéan A, Charlier A, Charrier F, Fares M, Le Bail M, Magrini MB, Savini I. 2013. Freins et leviers à la diversification des cultures. Étude au niveau des exploitations agricoles et des filières. Synthèse du rapport d'étude, INRA, $52 \mathrm{p}$.

Mourot J. 2009. Optimising the nutritional and sensorial profile of pork. Woodhead Publishing Ltd, Cambridge (Improving the sensory and nutritional quality of fresh meat).

Musella M, Cannata S, Rossi R, Mourot J, Baldini P, Corino C. 2009. Oméga-3 polyunsaturated fatty acid fromextruded linseed influences the fatty acid composition and sensory characteristics of dry-cured ham from heavy pigs. J. Anim. Sci. 87 : 3578-3588.

Noblet J, Jaguelin-Peyraud Y, Quemeneur B, Chesneau G. 2008. Valeur énergétique de la graine de lin chez le porc : impact de la technologie de cuisson-extrusion. 40 Journées de la Recherche Porcine. Paris, 5-6 février 2008, 203-208.

ONIDOL. 2011. L'avenir de la filière du Lin oléagineux français (Brochure). Paris, ONIDOL, $6 \mathrm{p}$.

Poncet C, Rémond D, Lepage E, Doreau M. 2003. Comment mieux valoriser les protéagineux et oléagineux enalimentation des ruminants Caractéristiques nutritionnelles des oléagineux (graines et tourteaux) et des protéagineux. Fourrages 174 : 205-229.

Razminowicz RH, Kreuzer M, Leuenberger H, Scheeder MRL. 2008. Efficiency of extruded linseed for the finishing ofgrass-fed steers to counteract a decline of oméga-3 fatty acids in the beef. Livestock Science 114 : 150-163.

Sauvant D, Perez JM, Tran G. 2004. Tables de composition et de valeurs nutritives des matières premières destinées auxanimaux d'élevage : porcs, volailles, bovins, ovins, caprins, lapins, chevaux, poissons. Inra Editions-AFZ, Versailles, 293 p.

SNIA. 2011. Les matières premières au coeur d'un métier. Brochure. $6 \mathrm{p}$.

Thévenin F. 2006. Oméga 3, l'harmonie des vaches et l'anti-stress de l'éleveur. L'avenir agricole et rural, Haute Marne (24 novembre 2006).

UNIP. 2012. Chiffres clés 2011-2012 Protéagineux France Pois Féverole - Lupin. UNIP, Paris, 2 p.

Weill P, Schmitt B, Legrand P. 2001. Évolution des paramètres lipidiques sanguins chez l'homme secondaire à l'introduction de lin, riche en acide alpha-linolénique (n-3), dans l'alimentation d'animaux destinés à la consommation humaine. OCL $8: 333-335$.

Weill P, Schmitt B, Chesneau G, Daniel N, Safraou F, Legrand P. 2002. Effects of introducing linseed in livestock dieton blood fatty acid composition of consumers of animal products. Ann. Nutr. Metab. 46 : 182-191.

Cite this article as: François Charrier, Marie-Benoît Magrini, Aude Charlier, M'hand Fares, Marianne Le Bail, Antoine Messéan, JeanMarc Meynard. Alimentation animale et organisation des filières : une comparaison pois protéagineux-lin oléagineux pour comprendre les facteurs freinant ou favorisant les cultures de diversification. OCL 2013, 20(4) D407. 


\section{Annexes}

\section{Taux d'incorporation des principales matières premières} dans les aliments composés

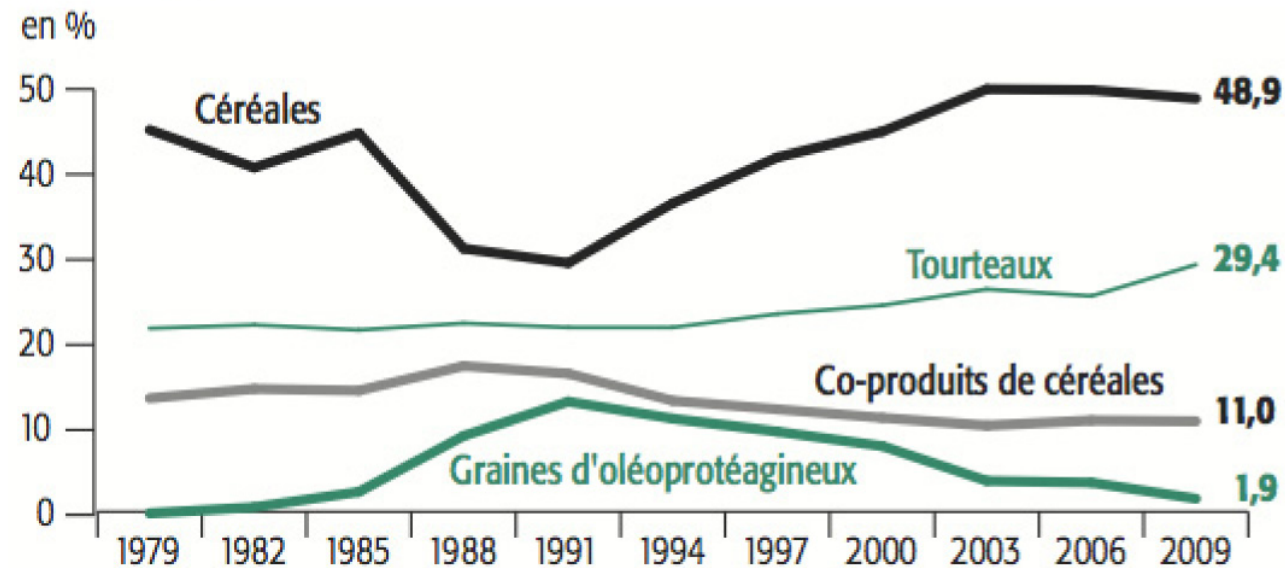

Fig. A.1. Taux d'incorporation des principales matières premières dans les aliments composés (Source : Agreste, 2011).

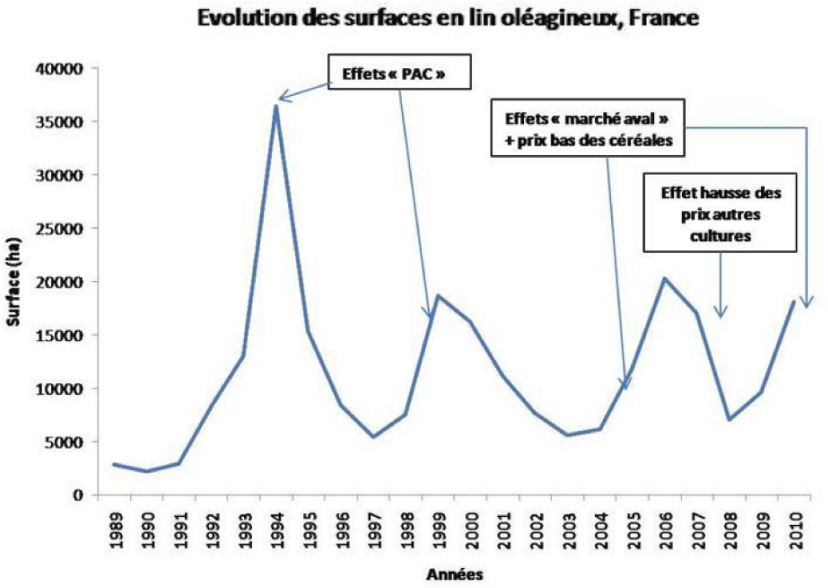

\begin{tabular}{|l|c|c|c|c|}
\hline & \multicolumn{2}{|c|}{ Pois protéagineux } & \multicolumn{2}{c|}{ Lin oléagineux } \\
\hline Année & 2011 & 2012 & 2011 & 2012 \\
\hline Surfaces (ha) & 183456 & 134115 & 16351 & 12080 \\
\hline $\begin{array}{l}\text { Rendement moyen } \\
\text { l00kg/ha) }\end{array}$ & 36 & 42 & 19 & 21 \\
\hline $\begin{array}{l}\text { Production } \\
\text { nationale (t) }\end{array}$ & 663387 & 556935 & 30547 & 25802 \\
\hline
\end{tabular}

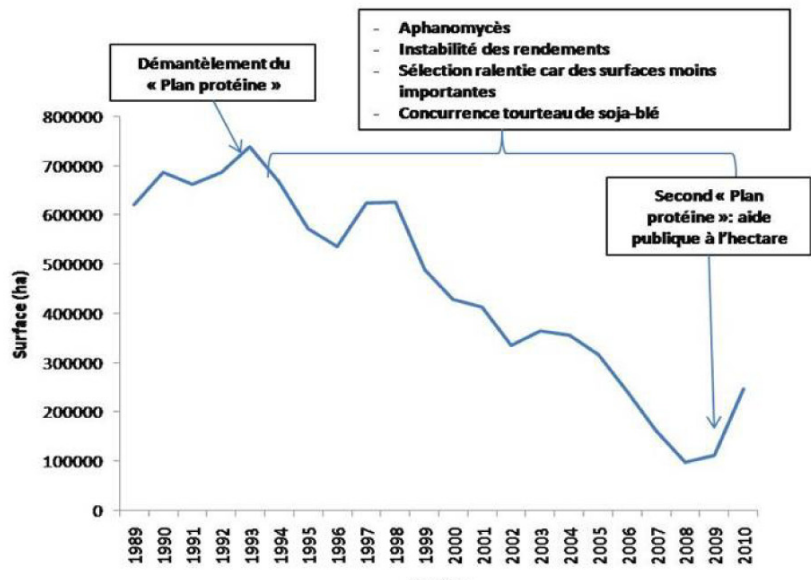

Fig. A.2. Chiffres clés sur le lin oléagineux et le pois protéagineux (Sources : UNIP, 2012; ONIDOL, 2011; Agreste, 2012). 